\title{
An experimental SDN proposal over legacy GPONs to allow real- time service and residential network reconfiguration
}

\author{
Noemí Merayo, David de Pintos, Juan Carlos Aguado, Ramón J. Durán, Ignacio de Miguel, Patricia \\ Fernández, Rubén M. Lorenzo, Evaristo J. Abril \\ Optical Communications Group, Department of Signal Theory, Communications and Telematic Engineering. \\ E.T.S.I. Telecomunicación, Universidad de Valladolid(Spain), Campus Miguel Delibes, Paseo de Belén 15, \\ 47011 Valladolid, Spain, \\ e-mail:noemer@tel.uva.es
}

\begin{abstract}
In this paper we propose an experimental SDN (Software Defined Networking) solution over legacy GPON (Gigabit Passive Optical Network) equipment that allow a control of the network configuration and its services. On the one hand, the proposal permits to move certain global bandwidth and service configuration policies outside the GPON so that they can be managed centrally by an SDN controller. In legacy PONs the real-time bandwidth allocation process is made inside the network infrastructure cycle by cycle between the OLT (Optical Line Terminal) and the ONTs (Optical Network Terminals) so the network performance could be adversely affected due to the latency between the SDN controller and the PON. In contrast, the control of some global DBA strategies by SDN techniques could lead to better network and management configuration and therefore our proposal is able to dynamically adjust these policies according to the real-time Quality of Service (QoS) requirements of residential users. On the other hand, the designed SDN proposal permits network subscribers to control the performance of their residential homes. In this way, they can set constraints and dynamically customize the bandwidth of their connected devices in a very transparent and efficient way.
\end{abstract}

Keywords: Software Defined Networking, Passive Optical Networks, OpenFlow, residential networks, Dynamic Bandwidth Allocation, Quality of Service.

\section{INTRODUCTION}

Passive Optical Networks (PONs) have become the most widely deployed access technology worldwide today. In fact, in the European Union 160 million households are connected by FTTH/B (Fiber To The Home/Building) technology. However, future predictions portend a greater increase in the next five years reaching 263 millions in 2025 [1]. Under this scenario, PON infrastructures are leading this market as it is expected that the $73 \%$ of FTTH infrastructures will be PON. Indeed, the GPON market worldwide is expected to reach USD 8.3 billion by 2025. On the other hand, there is an explosion of different connected devices and high demanded services that show critical Quality of Service (QoS) restrictions, so networks are suffering an extreme increase in their traffic. Therefore, it is highly necessary to provide next generation networks with intelligence, security and efficiency in its management and control. In this way, Software Defined Networking (SDN) can face these challenges and its integration in PON technologies is becoming increasingly important [2].

In SDN, the network control comes off the hardware and is given to a software application called SDN controller, so that the network can be intelligently and centrally controlled, using software applications and specific protocols, such as OpenFlow [3]. In order to provide this intelligent and centralized management, SDN separates the control plane of the network from the data plane that forwards the traffic inside the network devices. As a consequence, operators can manage, supervise and update the entire network regardless of the underlying network technology. Moreover, SDN can provide additional advantages in access networks, such as PONs. For example, it permits a fast control and reconfiguration of services and client applications of residential subscribers, or it permits to quickly detect network attacks and to efficiently apply automatized and specific responses to them. Furthermore, SDN can allow the coexistence of PON equipment of multiple vendors as the SDN protocols provide a common and unique programmable interface.

As a consequence, many SDN proposals are focusing in PONs. For example, many studies focus on turning legacy PON devices, OLTs (Optical Line Terminals) and ONTs (Optical Network Units), into SDN-controllable devices [4]. Other research studies are working on moving bandwidth allocation (DBA, Dynamic Bandwidth Allocation) and service configuration policies out of the PON intelligence, so that the SDN controller manages this network configuration in a centralized way [5]. Besides, as residential networks are becoming very complex due to the large number of connected devices and increasingly restrictive applications in quality of service requirements many proposals focus on SDN management techniques in residential networks [6]. Finally, other studies propose to develop virtual PONs [7] with SDN, where each virtual PON implements different configuration strategies such as bandwidth allocation policies. 
Therefore, we have developed an SDN management layer over legacy Gigabit Passive Optical Networks, so that network subscribers can manage restrictions in their home devices (smartphones, tablets, laptops) using an easy-to-use web application that permits them to contract services in real time, as well as to control their connected devices. Besides, the SDN management layer is able to configure some global DBA or service configuration strategies according to the real time QoS requirements of residential subscribers (ONTs).

\section{Definition of the SDN-GPON scenario}

The implemented SDN-GPON scenario allows operators/ISPs (Internet Service Providers) to configure a legacy GPON using an external SDN controller (OpenDayLight [8]) and several Open Virtual Switches (OVS) [9] using OpenFlow. As it can be observed in Fig. 1, in order to turn legacy OLTs and ONTs into OpenFlow controllable devices, we have implemented an SDN hardware abstraction layer, using Raspberry Pi's and computers, to embed the OVS's connected to the legacy equipment. The OVS connected to the OLT is called Centralized OVS (COVS) and the ones connected to the ONTs are called Remote OVS (ROVS). Then, the centralized ODL controller can modify the PON configuration and its services and user profiles in dynamic and real-time scenarios. In order to set the network configuration, the ODL controller sends flow tables to the COVS and ROVS to configure services (Internet, HDTV, VoIP) and these entries are real-time modified when residential users demand modifications in their QoS requirements. In fact, to configure one service (such as Internet) two flows are set: one for the downstream (COVS) and another for the upstream (ROVS) service requirements. Besides, in our proposal the QoS requirements are related to maximum bandwidth levels implemented with OpenFlow meters and associated with the corresponding flow entries. A meter measures the rate of packets assigned to one specific flow and enables controlling the rate of those packets so when this rate is larger than the value defined in the meter, packets are discarded. Therefore, a meter defines a rate limiter band, that is, the maximum downstream/upstream bandwidth.

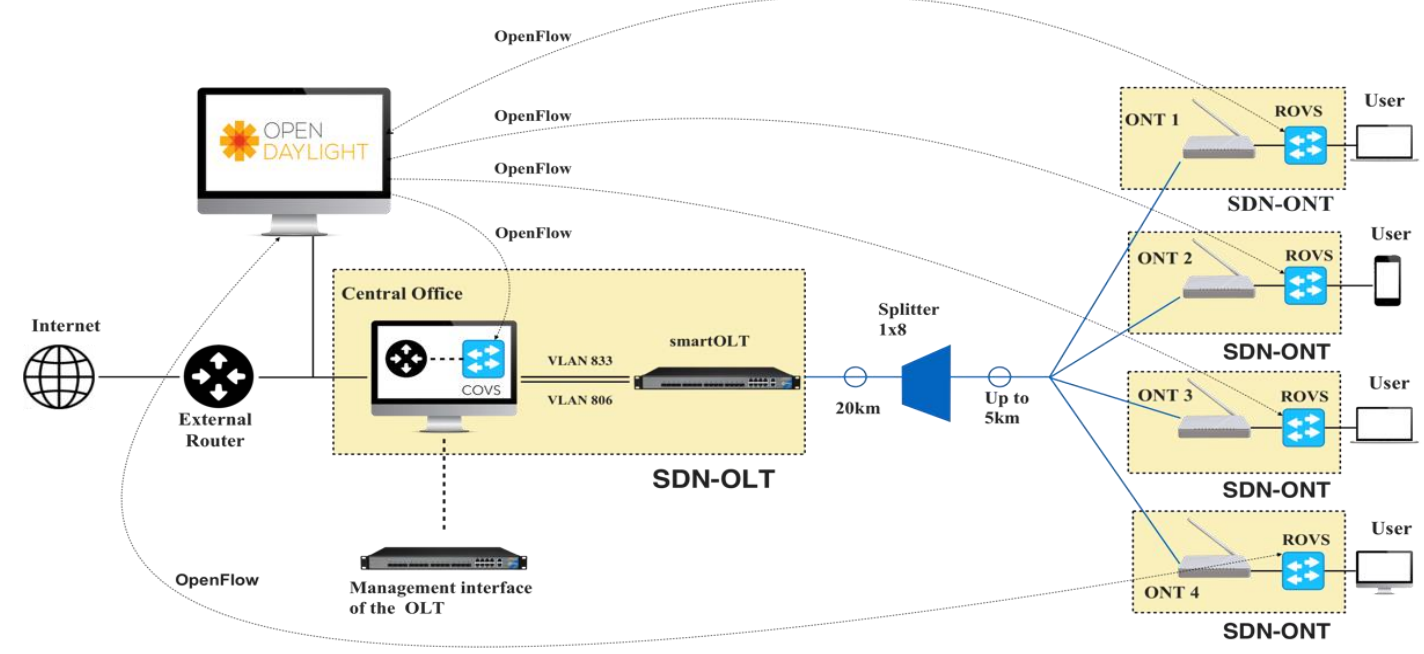

Figure 1. Description of the proposed SDN-PON scenario.

\section{Validation analysis and results of the QoS management proposal}

\subsection{Description of the GPON testbed}

We have implemented our SDN-GPON proposal over a GPON testbed (Fig. 2) using equipment from the TelnetRI vendor [10]. It includes an OLT SmartOLT 350 and several ONTs (Wave Access 512 model [39]) that follow the ITU-T G.984.x specifications.

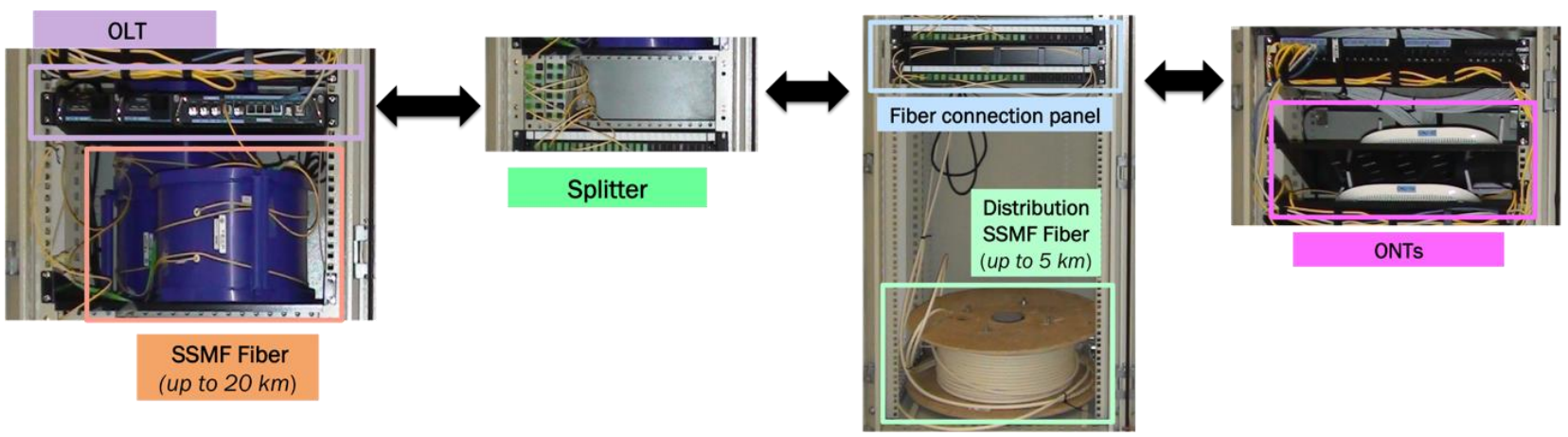

Figure 2. Description of GPON testbed. 
Moreover, the connection between the OLT and the splitter includes three spools of Standard Single Mode Fiber (SSMF) of different lengths, up to $20 \mathrm{~km}$. The splitter is connected to the ONTs by distribution fibers, so that each link can be individually configured, using a connection panel, from 100 meters up to $5 \mathrm{~km}$. Therefore, the GPON can emulate different distances from the residential users (ONTs) to the Central Office (OLT).

\subsection{Analysis of the Residential Network Management: Ad-Hoc Bandwidth Restriction}

We have programmed a web application that permits network subscribers to control their connected devices in the residential network (Fig. 3 (a)). In fact, they can restrict the maximum bandwidth associated with each connected device. In this example we consider a subscriber that contracts an Internet service of $100 \mathrm{Mbps}$ and several devices composing the residential network. Then, the residential subscriber wants to apply parental control over the "Son's smartphone" device restricting their bandwidth consumption. During the initial 3 minutes (180 s), the "Son's smartphone" device has a current restriction of 50 Mbps (Fig. 3 (a)), but the residential subscriber sets a new bandwidth restriction of $20 \mathrm{Mbps}$ for the next 3 minutes. Thus, Fig. 3 (b) represents the real time evolution of the assigned bandwidth using Wireshark for this device, and it can be observed that both restrictions are applied very fast and with low fluctuation in the transition.

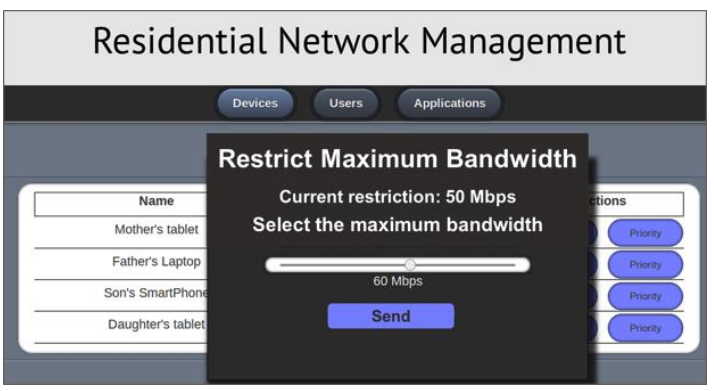

(a)

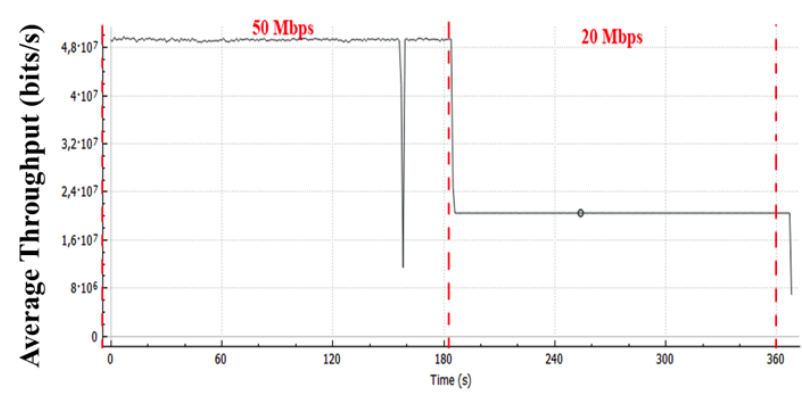

(b)

Figure 3. (a) Web application interface to apply bandwidth restrictions on devices (b) Real time evolution of the allocated bandwidth to the "Son's smartphone" device

\subsection{Analysis of external service configuration policies over the SDN-GPON proposal}

The developed SDN management layer implements some bandwidth configuration policies out of the OLT. Then, the ODL controller periodically monitor the demanded bandwidth of residential users (ONTs traffic) and it interchanges this information with the SDN management layer in other to apply algorithms to reconfigure the maximum bandwidth allocated to services. In this way, every OVS of the ONTs periodically sends packet statistics in OpenFlow messages, that is, packets processed and deleted by the meters. The SDN management layer is continually receiving this information from the SDN controller to calculate the real time demanded bandwidth using sliding windows to keep updated the demanded bandwidth of services of every ONT. At the same time, the management layer is executing a dynamic bandwidth management algorithm, so the algorithm monitors changes in the demanded bandwidth and it decides to increase or decrease the maximum bandwidth of services at a given time according to its contracted data rate. In this way, the algorithm can modify the maximum bandwidth of services by sending OpenFlow messages through the ODL controller.

To demonstrate the feasibility of our strategy we propose a new business model for service providers using flexible rates, so residential users contract a basic guaranteed bandwidth, but they may require an extra bandwidth at a special price. Then, we propose flexible rates differentiated by their basic bandwidth plus an excess bandwidth. If basic bandwidth is below $100 \mathrm{Mbps}$, the excess bandwidth is set $20 \%$ of the basic (basic rate), and if basic bandwidth is higher than $100 \mathrm{Mbps}$, a $30 \%$ of excess bandwidth can be given (premium rate).

To analyze the performance, we consider that a subscriber contracts a flexible basic rate of $100 \mathrm{Mbps}$ associated with an excess bandwidth of $20 \mathrm{Mbps}$ (20\%). Then, Fig. 4 (a) represents the real time demanded bandwidth along the time in Wireshark and Fig. 4 (b) the real time evolution of the allocated bandwidth according to the service configuration policy. In fact, it can be noticed in Fig. 4 (a) that the residential subscriber begins to demand $100 \mathrm{Mbps}$, and during the following 3 intervals of 3 minutes $(180 \mathrm{sec})$ it increases its demand to $110 \mathrm{Mbps}, 120 \mathrm{Mbps}$ and $140 \mathrm{Mbps}$, ending with a period of $100 \mathrm{Mbps}$. Furthermore, Fig. 4 (b) shows that the maximum offered bandwidth is $120 \mathrm{Mbps}$ that corresponds with its maximum permitted bandwidth (120 Mbps), even when the subscriber demands a rate of $140 \mathrm{Mbps}$ (fourth interval 540-720 s). For the remaining time intervals, the algorithm implemented in the SDN layer allocates the real-time required bandwidth since the demand is lower than the maximum of the contracted flexible rate, in a fast way and with stable response. 


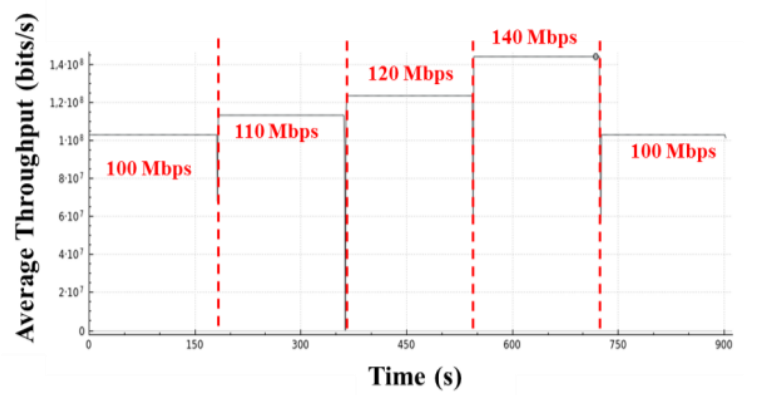

(a)

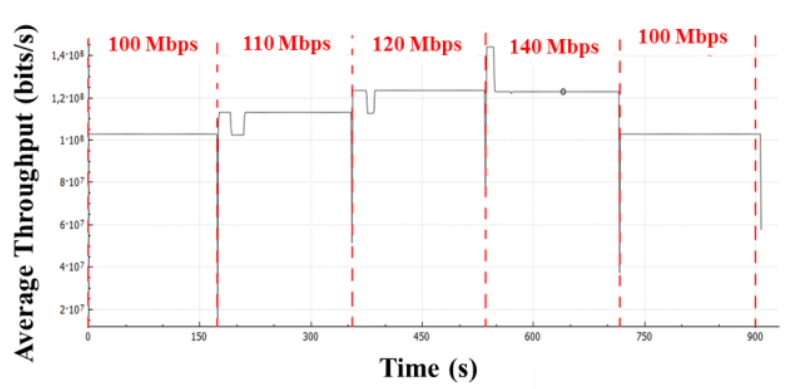

(b)

Figure 4. (a) Real time evolution of the demanded bandwidth for the basic flexible rate. (b) Real time evolution of the allocated bandwidth for the basic flexible rate

\section{CONCLUSIONS}

In this paper, we have described and demonstrated an SDN-GPON proposal on legacy equipment, which on the one hand allows network subscribers to dynamically control devices in their residential home as well as contract new services using a web application. On the other hand, the developed SDN management layer is able to take decisions regarding global DBA strategies by means of an external SDN controller to provide a global network management and configuration.

The proposed solution has been experimentally validated using OpenFlow and the OpenDayLight controller. Results have shown that residential users can impose bandwidth restrictions to their connected devices and the SDN system responds very quickly and the transitions and fluctuations to the new bandwidth levels are fast and low. Besides, when residential users contract flexible rates (basic bandwidth plus an extra), the SDN management layer efficiently applies service reconfiguration policies showing efficient and fast performance of the implemented strategy. Finally, our SDN-GPON proposal enables new business models for ISPs and network operators in which users can easily and efficiently control their residential networks and also it allows moving specific and global service configuration strategies out of the PON intelligence since the management layer can dynamically adjust this policies according to the real-time QoS requirements.

\section{ACKNOWLEDGEMENTS}

This work has been funded by Consejería de Educación de la Junta de Castilla y León (VA085G19), the Spanish Ministry of Economy and Competitiveness (TEC2017-84423-C3-1-P) and the INTERREG V-A España-Portugal (POCTEP) program (0677_DISRUPTIVE_2_E).

\section{REFERENCES}

[1] FTTH Council, "Europe Broadband status Market forecast by 20202025",https://www.ftthcouncil.eu/documents/Reports/2019/FTTH\%20Council\%20Europe\%20\%20Forecast\%20for\%20EUROPE\%202020-2025.pdf [Accessed: 10-April-2020].

[2] K. J. Kerpez, J. M. Cioffi, G. Ginis, M. Goldburg, S. Galli, and P. Silverman: Software-defined access networks, IEEE Communications Magazine, vol. 52, no. 9, pp. 152-159, Sept. 2014

[3] OpenFlow: Open Networking Foundation (ONF), in https://www.opennetworking.org/ [Accessed: 30September-2019.

[4] S. S. W. Lee, K.-Y. Li, and M.-S. Wu: Design and Implementation of a GPON-Based Virtual OpenFlowEnabled SDN Switch, IEEE/OSA Journal of Lightwave Technology, vol. 34, no. 10, pp. 2552-2561, May 2016.

[5] C. Qian et al. :Staged priority-based dynamic bandwidth allocation in software-defined hybrid passive optical network, Optical Engineering, vol. 57, no. 12, Dec. 2018.

[6] M. Amiri, H. Al Osman and S. Shimohammdi: SDN-enabled Game-Aware Network Management for Residential Gateways, in Proc. International Symposiym on Multimedia, Taichung, Taiwan, 2017, pp. 1-7.

[7] R. Challa, S.M. Raza, S. Jeon, H. Choo, and P. Thora: Refactoring Future Residential Networks into Slices, in Proc. IEEE Conference on NFV-SDN, Verona, Italy, 2018, pp. 1-7.

[8] OpenDayLight: The OpenDayLight SDN controller, in https://www.opendaylight.org/[Accessed: 01-April2019].

[9] Open vSwitch: Production Quality, Multilayer Open Virtual Switch, in https://www.openvswitch.org/, [Accessed: 10-March-2020].

[10] Telnet R-I: Telnet vendor web page, in https://www.telnet-ri.es/ [Accessed: 10-March-2020]. 